\title{
Cognitive Functions in Combat Posttraumatic Stress Disorder
}

\author{
Ana Havelka Meštrović ${ }^{1}$, Marina Domijan², Vlatko Mičkovićs, Zoran Lončar ${ }^{2}$ \\ ${ }^{1}$ Rochester Institute of Technology Croatia, Zagreb, Croatia \\ ${ }^{2}$ Clinic of Traumatology, Sisters of Mercy Clinical Hospital Center, Zagreb, Croatia \\ ${ }^{3}$ University of Applied Sciences, Zagreb, Croatia
}

\begin{abstract}
A B S T R A C T
Posttraumatic stress disorder (PTSD) is associated with changes in cognitive functions. The aim of the study was to investigate differences in cognitive abilities between PTSD patients and healthy controls. As PTSD is often accompanied by comorbidity, the PTSD patients with comorbid diagnoses were also included in our study. The study participants included 254 Croatian combat veterans (60 PTSD and 194 PTSD plus comorbidity) and control group of 125 healthy Croatian military and civilian pilots. The diagnosis of PTSD was made by clinical scale for PTSD assessment (CAPS), while cognitive abilities were measured by Wechsler intelligence scale (WAIS-III-R) and Rey test (ROCFT). The study results have confirmed that there is a significant difference in cognitive functions between the PTSD patients and healthy controls regarding age and education. The PTSD patients (PTSD only and PTSD with comorbidity) have shown lower general intellectual abilities, reduced capacity of working, numerical and visual memory, and decreased executive functions when compared to healthy controls. These results are an additional contribution to a better understanding and determination of changes in cognitive functions that occur in combat PTSD as a result of traumatic stress.
\end{abstract}

Key words: cognitive functions, PTSD, comorbidity, traumatic stress

\section{Introduction}

Posttraumatic stress disorder (PTSD) is defined as belonging to the group of anxiety disorders. It develops as a result of exposure to an individual's life and/or identity threatening traumatic events ${ }^{1}$. Traumatic experience can be the consequence of different events in previous life, e.g. childhood trauma, sexual abuse, natural disasters, or war induced trauma which is the basis of our study. With regard to the symptoms of PTSD, such as increased arousal, sleeping difficulties, nightmares, intrusive memory of traumatic events, frequent flashbacks, emotional numbness and irritability ${ }^{2}$, it may be concluded that PTSD significantly affects the functioning of an individual and his/ her quality of life. Changes in cognitive functioning are among the most common psychological consequences of PTSD that can add to the deterioration of an individual's general functioning. Individuals suffering from PTSD often have difficulties remembering information acquired a day or several hours ago. Thinking, memory, learning, motivational mechanisms, attitudes, beliefs, emotional content analysis - all of these form what is referred to as cognitive functions. The number of studies concerned with cognitive processes and PTSD has been on the increase in the past 30 years and they have significantly contributed to the development of cognitive psychology and neuroscience $^{3}$. Many neuropsychological studies have been carried out with PTSD patients so far ${ }^{4}$. A study on children population found differences in IQ between the abused children and the control group. It was concluded that the differences depended on the family and parental care and could not lead to the conclusion about any relation between PTSD and general intellectual functioning ${ }^{4}$. Studies carried out by Uddo et al. ${ }^{5}$ and others ${ }^{6}$ show general decrease in executive functions in PTSD patients, and also report about lower scores in working memory, reproduction of visual material and long-term memory. However, some studies $^{7}$ including the one by Barrett using the Wechsler intelligence measurement scale did not confirm the difference in working memory and general difficulties with memory in PTSD patients ${ }^{8}$. In the study on Gulf war veterans, Vasterling et al..$^{9}$ found difficulties in their working memory and increased probability for the development of retroactive interference. They used the Wisconsin Card Sorting Test (WCST) in this study and showed that the PTSD patients, unlike healthy controls with traumatic 
experience, did not exhibit deficits in declarative memory, but rather in attention and working memory tasks ${ }^{9}$. Despite many studies of cognitive functions and PTSD, there is no clear evidence of inter-relations between trauma and altered cognitive functioning. There are, however, indicators of trauma affecting cognitive abilities in terms of their changes as a result of its impact on the central and autonomous nervous system. However, previous neuropsychological studies were carried out with small number of neuropsychological tests. In our study a broad spectrum of congnitive tests was applied through one of the most widely used instruments for assessment of changes in cognitive functioning (WAIS-III-R). Furthermore, a group of PTSD patients with comorbid diagnoses was included in our study (e.g. depression, anxiety and somatoform disoders), and time elapsed since war events was shorter than in many previous studies.

The aim of our study was to investigate differences between different types of cognitive functions (verbal and non-verbal IQ, working and visual memory, executive functions) in individuals with combat PTSD with and without comorbid diagnoses in comparison with healthy controls.

\section{Materials and Methods}

\section{Participants}

The study comprised 254 Croatian war veterans (two clinical groups) treated at the Department of Psychiatry, Dubrava University Hospital in Zagreb. There were 60 PTSD patients without comorbid diagnoses and 194 PTSD patients with comorbidities, most often the anxiety and depressive disorders. The control group consisted of 125 Croatian military and civilian pilots undergoing systematic medical examinations at Dubrava University Hospital, Department of Aviation Medicine. The subjects of both PTSD groups were equalized in taking pharmacotherapeutic drugs (antidepressants, mood stabilizers and hypnotics), i.e. $84 \%$ were taking antidepressants, most commonly the SSRI and SSNI; 79\% were taking mood stabilizers; and $88 \%$ of study subjects were taking hypnotics. Since pharmacotherapy can affect the speed of motor and perceptive reactions, the speed of perception as a category of cognitive ability variable was excluded from further data analysis. Inclusion criteria: male combat veterans aged between 25-65 years, PTSD alone or with comorbid diagnoses according to CAPS results, war experience at least 3 months. Exclusion criteria: childhood history of trauma, family history of psychiatric disorders, neurodegenerative disorders, history of cognitive dysfunction or mental retardation, past or current alcohol or other substance abuse or addiction within 3 months, significant risk of violence or suicide. The patients with any type of physical disability were not included in the study sample. Table 1 presents the distribution of study subjects by socio-demographic characteristics, trauma features and CAPS results for the three study groups.

\section{Procedure}

The survey was conducted in the Department for Psychotrauma of the University Hospital Dubrava in Zagreb, from November 2006 to February 2010. Upon obtaining the written consent, all examinees were first subjected to the anamnesis questionnaire about sociodemographic status (age, education), previous and current disorders, traumatic experiences and combat-related experience, current symptoms, duration of symptoms, their intensity and treatment. PTSD and comorbid disorders were diagnosed by experienced psychiatrists using Mini Mental Neuropsychiatry Interview (MINI) ${ }^{\mathrm{a}}$ - a short structured clinical interview which enables researchers to make diagnoses of psychiatric disorders according to DSM-IV or ICD-10, and Clinician-Administered PTSD Scale (CAPS ${ }^{10}$. Clinical psychologists performed cognitive function measurements. Cognitive functions were measured with WAIS (Wechsler WAIS-Revised Version III) ${ }^{11}$. As regards the consumption of pharamacotherapeutic drugs, the perceptual speed as a measure of cognitive functions was excluded from our study results related to the group of PTSD patients for reasons of significant impact of pharmacotherapy on perceptive and motor speed. The measurement instruments used to assess our study results are highly reliable and valid, which is important in terms of diagnostic accuracy and determination of changes in cognitive functioning. The authors found high validity (internal consistency, $\alpha=.85$; and test-retest reliability, $r=.84$ ) for each of the three cluster symptoms (repeated flaschbacks, avoidance and increased arousal).

The WAIS-III-R is not standardized for Croatian population but it seems justified to use the standardization of other European countries (Germany - internal consistency $=.89$, and test-retest reliability, $\mathrm{r}=.91$; and UK - internal consistency $=.74$, and test-retest reliability, $r=.85$ ).

The data were analyzed by an experienced statistician. The data for war veterans with and without comorbid diagnoses and healthy control group on cognitive functions were matched using the t-test, and ANOVA at $95 \%$. Bonfferoni correction was used for assessing type 1 error (a- error). Groups results have shown normal distribution. Statistical analyses of the results included determination of descriptive parameters and comparison of the groups with respect to particular variables. Statistica 9.0 software package was used for data analysis.

Our hypothesis was that war veterans with PTSD and PTSD with comorbidity would have lower scores in cognitive functioning measures than healthy population.

\section{Results}

The obtained results show that there are differences in cognitive functions between PTSD patients and healthy controls. Statistically significant differences were confirmed for complete intelligence test, verbal and non-ver-

a http://harmresearch.org/index.php/mini-international-neuropsychiatric-interview-mini/ 
TABLE 1

DISTRIBUTION OF STUDY SUBJECTS BY SOCIO-DEMOGRAPHIC TRAITS, TRAUMA FEATURES AND CAPS RESULTS FOR THE THREE STUDY GROUPS

\begin{tabular}{|c|c|c|c|c|c|c|}
\hline Demographic characteristics & \multicolumn{2}{|c|}{$\begin{array}{c}\text { Group } 1 \\
\text { PTSD patients } \\
\quad(\mathrm{N}=60)\end{array}$} & \multicolumn{2}{|c|}{$\begin{array}{c}\text { Group } 2 \\
\text { PTSD + comorbidity } \\
(\mathrm{N}=194)\end{array}$} & \multicolumn{2}{|c|}{$\begin{array}{c}\text { Group } 3 \\
\text { Healthy controls } \\
(\mathrm{N}=134)\end{array}$} \\
\hline Age range & \multicolumn{2}{|c|}{$40-59$} & \multicolumn{2}{|c|}{$39-50$} & \multicolumn{2}{|c|}{$33-44$} \\
\hline Average age (M) & \multicolumn{2}{|c|}{48.7} & \multicolumn{2}{|c|}{41.4} & \multicolumn{2}{|c|}{35.5} \\
\hline Marital status & $\mathrm{N}$ & $\mathrm{f}(\%)$ & $\mathrm{N}$ & $\mathrm{f}(\%)$ & $\mathrm{N}$ & $\mathrm{f}(\%)$ \\
\hline Married & 23 & $38.3 \%$ & 58 & $43.3 \%$ & 72 & $37.1 \%$ \\
\hline Single & 4 & $6.7 \%$ & 36 & $26.9 \%$ & 6 & $3.1 \%$ \\
\hline Cohabitation & 17 & $28.3 \%$ & 30 & $22.4 \%$ & 83 & $42.8 \%$ \\
\hline Divorced & 16 & $26.7 \%$ & 10 & $7.5 \%$ & 33 & $17.0 \%$ \\
\hline Widowed & & 0 & \multicolumn{2}{|r|}{0} & & 0 \\
\hline Education & $\mathrm{N}$ & $\mathrm{f}(\%)$ & $\mathrm{N}$ & $\mathrm{f}(\%)$ & $\mathrm{N}$ & $\mathrm{f}(\%)$ \\
\hline Primary school & 16 & $26,7 \%$ & 69 & $35,6 \%$ & 11 & $8,2 \%$ \\
\hline Unfinished secondary school & 2 & $3,3 \%$ & 10 & $5,2 \%$ & 1 & $0.7 \%$ \\
\hline Secondary school & 36 & $60,0 \%$ & 92 & $47,4 \%$ & 76 & $56,7 \%$ \\
\hline Higher education (university degree) & 6 & $10,0 \%$ & 23 & $11,9 \%$ & 37 & $27,6 \%$ \\
\hline Master's or doctoral degree & 0 & $0 \%$ & 0 & $0 \%$ & 9 & $6,7 \%$ \\
\hline Working status & $\mathrm{N}$ & $\mathrm{f}(\%)$ & $\mathrm{N}$ & $\mathrm{f}(\%)$ & $\mathrm{N}$ & $\mathrm{f}(\%)$ \\
\hline Employed & 11 & $10,7 \%$ & 69 & $28,4 \%$ & 63 & $47,0 \%$ \\
\hline Unemployed & 34 & $22,0 \%$ & 102 & $59,3 \%$ & 71 & $53,0 \%$ \\
\hline Retired & 15 & $68,3 \%$ & 23 & $12,3 \%$ & 0 & $0 \%$ \\
\hline Years elapsed since traumatic event & \multicolumn{2}{|c|}{$\begin{array}{cc}\text { M } & \text { range of years } \\
13.8 & (13.5-14.7)\end{array}$} & $\mathrm{M}$ range of years & $\begin{array}{l}\text { ge of years } \\
(13.7-15.2)\end{array}$ & & \\
\hline Years of PTSD treatment & 9 & $(4.5-12.4)$ & 9.3 & $(5-12.7)$ & & \\
\hline Trauma characteristics & $\mathrm{N}$ & $\mathrm{f}(\%)$ & $\mathrm{N}$ & $f(\%)$ & $\mathrm{N}$ & $\mathrm{f}(\%)$ \\
\hline Participant or close witness to war events & 60 & $100 \%$ & 187 & $96 \%$ & & 0 \\
\hline Direct combat experience & 56 & $90.1 \%$ & 101 & $52 \%$ & & 0 \\
\hline Witnessing death of other people & 44 & $41.0 \%$ & 84 & $43 \%$ & & 0 \\
\hline Detention camp & 5 & $5.2 \%$ & 3 & $1.5 \%$ & & o \\
\hline$C A P S^{*}$ & \multicolumn{2}{|c|}{$\mathrm{M} \quad \mathrm{Sd}$} & \multicolumn{2}{|c|}{$\mathrm{M} \quad \mathrm{Sd}$} & \multicolumn{2}{|c|}{$\mathrm{M} \mathrm{Sd}$} \\
\hline Repeated flashbacks & \multicolumn{2}{|c|}{$65 \pm 9.5$} & \multicolumn{2}{|c|}{$62 \pm 8.4$} & \multicolumn{2}{|c|}{$12 \pm 2.5$} \\
\hline Evasion/Numbness & \multicolumn{2}{|c|}{$28 \pm 10.4$} & \multicolumn{2}{|c|}{$37 \pm 10.2$} & \multicolumn{2}{|c|}{$14 \pm 4.2$} \\
\hline Increased arousal & \multicolumn{2}{|c|}{$19 \pm 12.3$} & \multicolumn{2}{|c|}{$20 \pm 6.4$} & \multicolumn{2}{|c|}{$11 \pm 5.4$} \\
\hline Total & \multicolumn{2}{|c|}{$107 \pm 14.4$} & \multicolumn{2}{|c|}{$115 \pm 10.4$} & \multicolumn{2}{|c|}{$25 \pm 7.3$} \\
\hline
\end{tabular}

${ }^{*}$ CAPS - Clinical Administered Posttraumatic Stress Scale

bal intelligence $\left(\mathrm{F}_{\mathrm{iq}}=57.16 ; \mathrm{p}<0.01 ; \mathrm{F}_{\mathrm{viq}}=33.72 ; \mathrm{p}<0.01\right.$; $\mathrm{F}_{\text {niq }}=40.09 ; \mathrm{p}<0.01$ ), and for all subscales of WAIS-III-R test between the PTSD patients with and without comorbidities and heathy controls $\left(\mathrm{F}_{\text {memory }}=16.00 ; \mathrm{p}<0.01 ; \mathrm{F}_{\text {digit- }}\right.$ span $\left.=21.07 ; \mathrm{p}<0.01 ; \mathrm{F}_{\text {executive functions }}=70.01 ; \mathrm{p}<0.01\right)$. The results are shown in Table 2. Post hoc comparison of arithmetic means of the results for the three study groups was made by Fisher LSD test; it showed statistically significant differences $(\mathrm{p}<0.01)$ between the PTSD patients and healthy controls, and also between the PTSD patients with comorbid diagnoses and healthy controls in all subscales of the intelligence test and in the total verbal and non-verbal IQ (Table 2).
However, in some cases the principal effects of clinical status were modified by significant interactions (Table 2). As regards the subscales of verbal intelligence, a statistically significant difference was obtained between the patients with PTSD diagnosis only and the PTSD patients with comorbidities (Table 2). PTSD only group shows lower scores than control group in VIQ scale. In other subscales no statistically significant difference was obtained between the two PTSD study groups. A post hoc analysis of difference regarding education indicates significant difference between the study subjects with lower and those with higher education, particularly in the subscales of IQ, memory, working memory and matrix rea- 
TABLE 2

RESULTS OF MULTIVARIATE ANALYSIS OF VARIANCE OF COGNITIVE FUNCTIONING ON WAIS-III-R TEST FOR STUDY PARTICIPANTS OF DIFFERENT CLINICAL STATUS, AGE AND EDUCATION

\begin{tabular}{|c|c|c|c|c|}
\hline & $\mathrm{df}$ & $\mathrm{F}$ & $\mathrm{p}$ & Post hoc \\
\hline \multicolumn{5}{|l|}{ IQ } \\
\hline Group effect & 2 & 57.16 & $\mathrm{p}<0.01$ & \multirow[t]{3}{*}{$1-3^{*}, 2-3^{*}$} \\
\hline Age effect & 1 & 0.21 & $\mathrm{p}>0.01$ & \\
\hline Education effect & 2 & 1.70 & $\mathrm{p}>0.01$ & \\
\hline Interaction group*age & 2 & 0.70 & $\mathrm{p}>0.01$ & \multirow{4}{*}{$\begin{array}{c}1-3^{*}, 2-3^{*} \\
\text { ES-C }\end{array}$} \\
\hline Interaction group*education & 4 & 2.67 & $\mathrm{p}<0.01$ & \\
\hline Interaction group*age*education & 4 & 1.28 & $\mathrm{p}>0.01$ & \\
\hline \multicolumn{4}{|l|}{ VIQ } & \\
\hline Group effect & 2 & 33.72 & $\mathrm{p}<0.01$ & \multirow[t]{6}{*}{$1-2^{*}, 1-3^{*}, 2-3^{*}$} \\
\hline Age effect & 1 & 0.54 & $\mathrm{p}>0.01$ & \\
\hline Education effect & 2 & 0.61 & $\mathrm{p}>0.01$ & \\
\hline Interaction group*age & 2 & 0.39 & $\mathrm{p}>0.01$ & \\
\hline Interaction group*education & 4 & 2.34 & $\mathrm{p}>0.01$ & \\
\hline Interaction group*age*education & 4 & 0.28 & $\mathrm{p}>0.01$ & \\
\hline \multicolumn{5}{|l|}{ NIQ } \\
\hline Group effect & 2 & 40.09 & $\mathrm{p}<0.01$ & \multirow[t]{6}{*}{$1-3^{*}, 2-3^{*}$} \\
\hline Age effect & 1 & 0.88 & $\mathrm{p}>0.01$ & \\
\hline Education effect & 2 & 0.94 & $\mathrm{p}>0.01$ & \\
\hline Interaction group*age & 2 & 1.11 & $\mathrm{p}>0.01$ & \\
\hline Interaction group*education & 4 & 1.87 & $\mathrm{p}>0.01$ & \\
\hline Interaction group*age*education & 4 & 1.28 & $\mathrm{p}>0.01$ & \\
\hline \multicolumn{5}{|l|}{ INFORMATION } \\
\hline Group effect & 2 & 27.37 & $\mathrm{p}<0.01$ & \multirow[t]{6}{*}{$1-3^{*}, 2-3^{*}$} \\
\hline Age effect & 1 & 0.30 & $\mathrm{p}>0.01$ & \\
\hline Education effect & 2 & 0.50 & $\mathrm{p}>0.01$ & \\
\hline Interaction group*age & 2 & 0.13 & $\mathrm{p}>0.01$ & \\
\hline Interaction group*education & 4 & 1.78 & $\mathrm{p}>0.01$ & \\
\hline Interaction group*age*education & 4 & 0.35 & $\mathrm{p}>0.01$ & \\
\hline \multicolumn{5}{|l|}{ COMPREHENSION } \\
\hline Group effect & 2 & 18.20 & $\mathrm{p}<0.01$ & \multirow[t]{6}{*}{$1-3^{*}, 2-3^{*}$} \\
\hline Age effect & 1 & 1.89 & $\mathrm{p}>0.01$ & \\
\hline Education effect & 2 & 0.47 & $\mathrm{p}>0.01$ & \\
\hline Interaction group*age & 2 & 1.27 & $\mathrm{p}>0.01$ & \\
\hline Interaction group*education & 2 & 0.72 & $\mathrm{p}>0.01$ & \\
\hline Interaction group*age*education & 4 & 0.42 & $\mathrm{p}>0.01$ & \\
\hline \multicolumn{5}{|l|}{ MEMORY } \\
\hline Group effect & 2 & 16.00 & $\mathrm{p}<0.01$ & \multirow[t]{5}{*}{$1-3^{*}, 2-3^{*}$} \\
\hline Age effect & 1 & 0.12 & $\mathrm{p}>0.01$ & \\
\hline Education effect & 2 & 0.15 & $\mathrm{p}>0.01$ & \\
\hline Interaction group*age & 2 & 1.95 & $\mathrm{p}>0.01$ & \\
\hline Interaction group*education & 4 & 2.05 & $\mathrm{p}>0.01$ & \\
\hline \multirow[t]{2}{*}{ Interaction group*age*education } & 4 & 2.49 & $\mathrm{p}<0.01$ & $1-3^{*}, 2-3^{*}$ \\
\hline & & & & ES-C*, Y-O* \\
\hline \multicolumn{5}{|l|}{ MEMORY DIGIT SPAN FORWARD } \\
\hline Group effect & 2 & 21.07 & $\mathrm{p}<0.01$ & \multirow[t]{2}{*}{$1-3^{*}, 2-3^{*}$} \\
\hline Age effect & 1 & 3.12 & $\mathrm{p}>0.01$ & \\
\hline
\end{tabular}




\begin{tabular}{|c|c|c|c|c|}
\hline & df & $\mathrm{F}$ & $\mathrm{p}$ & Post hoc \\
\hline Education effect & 2 & 0.15 & $\mathrm{p}>0.01$ & \\
\hline Interaction group*age & 2 & 2.51 & $\mathrm{p}>0.01$ & \\
\hline Interaction group*education & 4 & 1.64 & $\mathrm{p}>0.01$ & \\
\hline Interaction group*age*education & 4 & 2.33 & $\mathrm{p}>0.01$ & \\
\hline \multicolumn{5}{|c|}{ MEMORY DIGIT SPAN BACKWARD } \\
\hline Group effect & 2 & 70.01 & $\mathrm{p}<0.01$ & $1-3^{*}, 2-3^{*}$ \\
\hline Age effect & 1 & 1.18 & $\mathrm{p}>0.01$ & \\
\hline Education effect & 2 & 1.34 & $\mathrm{p}>0.01$ & \\
\hline Interaction group*age & 2 & 0.91 & $\mathrm{p}>0.01$ & \\
\hline Interaction group*education & 4 & 2.93 & $\mathrm{p}<0.01$ & $1-3^{*}, 2-3^{*}, \mathrm{ES}-\mathrm{C}^{*}$ \\
\hline Interaction group*age*education & 4 & 2.66 & $\mathrm{p}<0.01$ & $1-3^{*}, 2-3^{*}, \mathrm{ES}-\mathrm{C}^{*}, \mathrm{Y}-\mathrm{O} *$ \\
\hline \multicolumn{5}{|l|}{ ARITHMETIC } \\
\hline Group effect & 2 & 30.15 & $\mathrm{p}<0.01$ & $1-3^{*}, 2-3^{*}$ \\
\hline Age effect & 1 & 0.47 & $\mathrm{p}>0.01$ & \\
\hline Education effect & 2 & 2.15 & $\mathrm{p}>0.01$ & \\
\hline Interaction group*age & 2 & 0.31 & $\mathrm{p}>0.01$ & \\
\hline Interaction group*education & 4 & 1.72 & $\mathrm{p}>0.01$ & \\
\hline Interaction group*age*education & 4 & 1.26 & $\mathrm{p}>0.01$ & \\
\hline \multicolumn{5}{|l|}{ SIMILARITIES } \\
\hline Group effect & 2 & 5.19 & $\mathrm{p}<0.01$ & $1-3^{*}, 2-3^{*}$ \\
\hline Age effect & 1 & 0.71 & $\mathrm{p}>0.01$ & \\
\hline Education effect & 2 & 1.00 & $\mathrm{p}>0.01$ & \\
\hline Interaction group*age & 2 & 2.72 & $\mathrm{p}>0.01$ & \\
\hline Interaction group*education & 4 & 1.01 & $\mathrm{p}>0.01$ & \\
\hline Interaction group*age*education & 4 & 1.17 & $\mathrm{p}>0.01$ & \\
\hline \multicolumn{5}{|l|}{ MATRIX REASONING } \\
\hline Group effect & 2 & 41.76 & $\mathrm{p}<0.01$ & $1-3^{*}, 2-3^{*}$ \\
\hline Age effect & 1 & 0.45 & $p>0.01$ & \\
\hline Education effect & 2 & 3.05 & $\mathrm{p}>0.01$ & \\
\hline Interaction group*age & 2 & 0.10 & $\mathrm{p}>0.01$ & \\
\hline Interaction group*education & 4 & 3.38 & $\mathrm{p}<0.01$ & $1-3^{*}, 2-3^{*}, \mathrm{ES}-\mathrm{C}^{*}$ \\
\hline Interaction group*age*education & 4 & 0.92 & $\mathrm{p}>0.01$ & \\
\hline \multicolumn{5}{|l|}{ PICTURE COMPLETION } \\
\hline Group effect & 2 & 37.92 & $\mathrm{p}<0.01$ & $1-3^{*}, 2-3^{*}$ \\
\hline Age effect & 1 & 1.84 & $\mathrm{p}>0.01$ & \\
\hline Education effect & 2 & 0.21 & $\mathrm{p}>0.01$ & \\
\hline Interaction group*age & 2 & 1.17 & $\mathrm{p}>0.01$ & \\
\hline Interaction group*education & 4 & 0.94 & $\mathrm{p}>0.01$ & \\
\hline Interaction group*age*education & 4 & 1.95 & $\mathrm{p}>0.01$ & \\
\hline \multicolumn{5}{|l|}{ BLOCK DESIGN } \\
\hline Group effect & 2 & 0.99 & $\mathrm{p}<0.01$ & $1-3^{*}, 2-3^{*}$ \\
\hline Age effect & 1 & 64.40 & $\mathrm{p}>0.01$ & \\
\hline Education effect & 2 & 1.95 & $\mathrm{p}>0.01$ & \\
\hline Interaction group*age & 2 & 0.94 & $\mathrm{p}>0.01$ & \\
\hline Interaction group*education & 4 & 2.31 & $\mathrm{p}>0.01$ & \\
\hline Interaction group*age*education & 4 & 0.99 & $\mathrm{p}>0.01$ & \\
\hline \multicolumn{5}{|l|}{ SYMBOL SEARCH } \\
\hline Group effect & 2 & 79.84 & $\mathrm{p}<0.01$ & $1-3^{*}, 2-3^{*}$ \\
\hline Age effect & 1 & 2.06 & $\mathrm{p}>0.01$ & \\
\hline
\end{tabular}




\begin{tabular}{lrrrc}
\hline & df & $\mathrm{F}$ & $\mathrm{p}$ & Post hoc \\
\hline Education effect & 2 & 0.76 & $\mathrm{p}>0.01$ & \\
Interaction group*age & 2 & 0.37 & $\mathrm{p}>0.01$ & \\
Interaction group*education & 4 & 1.67 & $\mathrm{p}>0.01$ & \\
Interaction group*age*education & 4 & 2.47 & $\mathrm{p}>0.01$ & \\
CODING & & & & \\
Group effect & 2 & 69.84 & $\mathrm{p}<0.01$ & $1-3^{*}, 2-3^{*}$ \\
Age effect & 1 & 3.06 & $\mathrm{p}>0.01$ & \\
Education effect & 2 & 1.87 & $\mathrm{p}>0.01$ & \\
Interaction group*age & 2 & 3.26 & $\mathrm{p}<0.01$ & ES-C*, Y-O* \\
Interaction group*education & 4 & 2.91 & $\mathrm{p}<0.01$ & $1-3^{*}, 2-3^{*}$, ES-C \\
Interaction group*age*education & 4 & 2.47 & $\mathrm{p}<0.01$ & $1-3^{*}, 2-3^{*}$, ES-C \\
\hline Group 1 (PTSD), Group 2 (PTSD and comorbidity), Group 3 (control group), ES (elemen- \\
tary school), HS (high school), C (Colledge or Faculty degree), Younger age - 33-45, Older \\
age - 46-59
\end{tabular}

soning; a significant difference between those with high school education and those with higher education in subscale results can be seen for the variables of numeric working memory and matrix reasoning, whereas no difference was observed between those with only primary and those with secondary school education for any of the subscales. A statistically significant difference between those with primary and those with higher education level was obtained in the overall IQ scale as well as in verbal and non-verbal intelligence scales (Table 2). Regarding education and age, there were no differences between the PTSD patients and healthy controls in the group of younger age and higher education status in memory scales. However, the PTSD patients with comorbidity, at the same age and education level, showed significantly lower results on memory scales.

No significant differences were found for the interaction effects of age, education and clinical status in visual memory (short and long-term), speed of problem solving and total result for visual and spatial abilities (Table 3). Significant differences between the study groups were obtained, and the post hoc analysis additionally confirmed their statistical significance, between the clinical groups of PTSD patients with and without comorbidities in comaprison with healthy controls, i.e. the comparable group on ROCFT $\left(\mathrm{F}_{\text {ROCFT }}=8.18 ; \mathrm{p}<0.05 ; \mathrm{F}_{\text {vis.memory }}=39.03 ; \mathrm{p}<0.05\right.$; $\mathrm{F}_{\text {vis.memory-long-term }}=27.73 ; \mathrm{p}<0.05$ ) (Table 3 ).

\section{Discussion}

Our study results showed significantly poorer results in total cognitive functioning of PTSD patients with and without comorbidities (depression, GAD, somatoform disorder) particularly in the domains of working memory and executive functioning. The results about the changes in intellectual capacity, lowered verbal, non-verbal and general intellectual capacities in PTSD patients confirm that there are differences in verbal and non-verbal intelligence in PTSD patients when compared with healthy controls (Table 2). These results are in line with previous studies on veter- ans ${ }^{12}$. Similar to our study results, many previous studies confirm diminishing of cognitive capacity that occurs as a consequence of traumatic experience, even if the trauma is not associated with war events. However, some studies did not find any differences on intelligence score between PTSD and non-PTSD groups, which is opposite to our study results $^{13}$. Regarding specific cognitive deficits, especially in the domain of attention, working memory (ability to temporarily maintain and manipulate information) and executive functions (scores on scales - arithmetic, block design, digit span forward, digit span backward) our study shows significantly lower results in the PTSD patients with and without comorbidity as compared to healthy controls. Some previously conducted neuropsychological studies report similar results, although with a smaller number of study subjects, longer time elapsed since war events, no control group with comorbidities, and shorter measurement instruments ${ }^{14}$. Our results are in line with many previous studies which have found that PTSD can decrease memory and executive functions ${ }^{15,16}$. Previous findings suggest that persons with PTSD experience impairments in their working memory and processing tasks which are related to a damage of the orbitofrontal and amygdala regions, and their numerous projections ${ }^{16}$. The role of amygdala in anxiety disorders may mean that the cognitive function is impaired by the disruptive influence of a heightened arousal and not by the actual damage of that area ${ }^{17}$. Our findings about the impairments of cognitive functions are a significant contribution to a better understanding of cognitive changes that occur after war trauma PTSD because of our study sample (control of education status and age, pharmacotherapy and comorbidities).

It has often been the matter of dispute whether cognitive difficulties are related to PTSD or perhaps to some other comorbid diagnosis, e.g. depression or anxiety. Previous investigations of PTSD in Croatia show that PTSD patients often have comorbid diagnoses which can also influence cognitive functioning ${ }^{18}$. Our results show that patients with PTSD and comorbidity scored the lowest results in cognitive functioning when compared with those with only PTSD and healthy controls. As to PTSD patients without comor- 
TABLE 3

RESULTS OF MULTIVARIATE ANALYSIS OF VARIANCE OF VISUAL MEMORY AND SPATIAL FUNCTIONS (ROCFT) FOR STUDY PARTICIPANTS OF DIFFERENT CLINICAL STATUS, AGE AND EDUCATION

\begin{tabular}{|c|c|c|c|c|}
\hline & df & $\mathrm{F}$ & $\mathrm{p}$ & Post hoc \\
\hline \multicolumn{5}{|l|}{ SCORE ROCFT } \\
\hline Group effect & 2 & 8.18 & $\mathrm{p}<0.05$ & \multirow[t]{6}{*}{$1-3^{*}, 2-3^{*}$} \\
\hline Age effect & 1 & 3.24 & $\mathrm{p}>0.05$ & \\
\hline Education effect & 2 & 1.58 & $\mathrm{p}>0.05$ & \\
\hline Interaction group*age & 2 & 2.59 & $\mathrm{p}>0.05$ & \\
\hline Interaction group*education & 4 & 1.95 & $\mathrm{p}>0.05$ & \\
\hline Interaction group*age*education & 4 & 0.65 & $\mathrm{p}>0.05$ & \\
\hline \multicolumn{5}{|l|}{ SHORT TERM VISUAL MEMORY } \\
\hline Group effect & 2 & 39.03 & $\mathrm{p}<0.05$ & \multirow[t]{6}{*}{$1-3^{*}, 2-3^{*}$} \\
\hline Age effect & 1 & 0.85 & $\mathrm{p}>0.05$ & \\
\hline Education effect & 2 & 0.54 & $\mathrm{p}>0.05$ & \\
\hline Interaction group*age & 2 & 0.31 & $\mathrm{p}>0.05$ & \\
\hline Interaction group*education & 4 & 0.35 & $\mathrm{p}>0.05$ & \\
\hline Interaction group*age*education & 4 & 0.60 & $\mathrm{p}>0.05$ & \\
\hline \multicolumn{5}{|l|}{ LONG TERM VISUAL MEMORY } \\
\hline Group effect & 2 & 27.73 & $\mathrm{p}<0.05$ & \multirow[t]{6}{*}{$1-3^{*}, 2-3^{*}$} \\
\hline Age effect & 1 & 1.23 & $\mathrm{p}>0.05$ & \\
\hline Education effect & 2 & 1.74 & $\mathrm{p}>0.05$ & \\
\hline Interaction group*age & 2 & 1.17 & $\mathrm{p}>0.05$ & \\
\hline Interaction group*education & 4 & 1.21 & $\mathrm{p}>0.05$ & \\
\hline Interaction group*age*education & 4 & 0.13 & $\mathrm{p}>0.05$ & \\
\hline \multicolumn{5}{|l|}{ MOTORIC SPEED } \\
\hline Group effect & 2 & 81.34 & $\mathrm{p}<0.05$ & \multirow[t]{6}{*}{$1-3^{*}, 2-3^{*}$} \\
\hline Age effect & 1 & 1.55 & $\mathrm{p}>0.05$ & \\
\hline Education effect & 2 & 0.16 & $\mathrm{p}>0.05$ & \\
\hline Interaction group*age & 2 & 1.78 & $\mathrm{p}>0.05$ & \\
\hline Interaction group*education & 4 & 2.04 & $\mathrm{p}>0.05$ & \\
\hline Interaction group*age*education & 4 & 1.09 & $\mathrm{p}>0.05$ & \\
\hline
\end{tabular}

bidity, we found statistically significant differences only in verbal intelligence scale, while again PTSD patients with comorbidity showed poorer results when compared to those with PTSD alone (Table 2). Also, this is the only scale showing a difference between PTSD patients without and PTSD patients with comorbidities. Such results have not yet been confirmed by other studies in which differences were obtained only in verbal memory between PTSD and non PTSD subjects ${ }^{5}$. Verbal memory can be related to comorbidities especially to anxiety disorders, the same as verbal intelligence when measured by WAIS-III-R. It is also possible that, in addition to trauma, general verbal factor can be affected by comorbid disorders as well. Changes in the scale of overall verbal intelligence could be considered as a specific result of our study since they have not been confirmed by any previous study on PTSD patients with and without comorbid diagnoses so far.
Apart from reasons related to our study design, the results obtained could be explained by the fact that our study subjects were war veterans who were not professional soldiers. There are differences in personality traits of individuals who are professional soldiers properly prepared and trained for war activities vs. individuals who joined war actions from their civilian lives (the so-called war-volunteers). It should also be taken into consideration that our study subjects worried deeply about their families left in areas exposed to war, which additionally aggravated their otherwise most stressful situations and thus contributed to a greater intensity of their traumatic experiences and number of comorbid conditions ${ }^{19,20}$.

The PTSD patients with comorbidities showed the poorest results in all other scales. In a previous study on Croatian combat veterans similar results were obtained and PTSD patients with comorbidities showed lower 
scores in cognitive functions when compared with patients with PTSD alone and healthy individuals. Most of previous studies about cognition and war PTSD did not include PTSD patients with comorbid diagnoses and they applied shorter set of neuropsychological tests for the measurement of cognitive functions. Our study results are in accordance with those obtained by Barrett et al., who included the PTSD patients with comorbid diagnoses (MDD, GAD, Substance abuse disorders) ${ }^{8}$. Despite similarity in the obtained results, our study did not include patients with substance abuse history, since it could influence cognitive ability test results. We also controlled the influence of pharmacotherapy on cognitive functions. The results showed no significant difference in cognitive functioning within the group of patients with comorbidities (i.e. there is no difference related to GAD, depression and somatoform disorders) (Table 2).

Knowing that education level and age can be a significant factor in affecting cognitive functions ${ }^{14}$, these variables were also taken into consideration. Our results show that when age, education, and PTSD patients with comorbid diagnoses were included, significant difficulties in cognitive functioning of PTSD patients with and without comorbidities were found when compared with healthy population (Table 2). We found that younger individuals and those with higher education scored highest in memory and executive functions, and also in motor speed and total intelligence score when compared with older individuals and those of lower educational status, regardless of their clinical conditions. A significant difference was also confirmed with regard to the capacity of working memory. The obtained results suggest how important it is to include the variables of age and education when studying cognitive functions. No significant difference was observed in the capacities of working memory, i.e. executive functions in PTSD patients and healthy controls in the group of highly educated study subjects. The PTSD patients (younger individuals with higher education) showed slightly better results in the memory scale. The finding may substantiate the thesis that higher education may have to a certain degree a protective role in the development of $\mathrm{PTSD}^{9,21}$, particularly in younger individuals i.e. that in these individuals the decline in cognitive abilities will be less expressed ${ }^{22}$.

The obtained results further show that education is significant only in younger individuals, whereas older adults with higher education show significantly poorer results when compared with healthy population. The previous studies including combat veterans did not control the variable of age, since in majority of them the veterans were of older age. Our study shows that the PTSD patients with comorbidities achieve lower results regardless of age and education. Hence, it is also possible that, if a person with PTSD has developed depression or anxiety as a comorbid diagnosis, education and age do not play a significant role in changes of cognitive abilities.

There has been little research of visual memory in individuals with PTSD. Our results regarding visual and spatial abilities show that PTSD patients achieve significantly lower results in visual-spatial organization and visual memory in all age groups and education levels (Table 3). These results are opposed to those obtained by a number of previous studies indicating that the visual and spatial perception remain preserved in PTSD patient $^{23,24}$. The study of Gilbertson et al. ${ }^{12}$ is the only study, apart from ours, confirming changes in all aspects of visual and spatial perception or functioning, although it is a general opinion that their sample was influenced by peritraumatic experiences, thus rendering these results less reliable (valuable). However, these findings of impairments in visual memory and deficits in visual-spatial perception should not be ignored.

Limitations of our study may be attributed to biased trauma experience and the sample which included males only, mostly because the number of women directly involved in combat situations was too small. Furthermore, during the time of our study some PTSD patients were undergoing procedure of solving their material claims based on their physical and mental damages, which might have led to simulation of results ${ }^{26}$.

In summary, our study results show that there are differences in cognitive abilities between individuals with traumatic war experience and those without it, particularly regarding the PTSD patients with comorbid diagnoses who achieve significantly lower results in cognitive abilities tests.

According to research results we can conclude that the individuals suffering from post-war traumatic disorder (i.e. PTSD), show lower general intelligence level than healthy individuals of all age groups; the greatest difference may be noticed in the group of highly educated persons. It has also been confirmed that combat PTSD patients have lower verbal and non-verbal intelligence, and lower visual memory when compared with healthy controls. Older combat PTSD patients with higher education have lower working memory and executive functions when compared with the comparable group of the same age and education level. Younger PTSD patients with higher education do not significantly differ in working memory and executive functions from healthy individuals of the same age and education.

Future study designs should be aimed at the possibilities of defining pre-morbid functioning of PTSD individuals, their sex and social differences, and the differences in traumatic experiences when measuring changes and differences in cognitive abilities. It is also important to monitor social rehabilitation of the diseased and their quality of life that might affect cognitive changes.

\section{Acknowledgement}

The research was conducted within the project „Psychophysiology of stress related disorders“, Principal Investigator: Dragica Kozaric-Kovacic, funded by the Croatian Ministry of Science, Education and Sport. 


\section{R E F E R E N C E S}

1. AMERICAN PSYCHIATRIC ASSOCIATION. Diagnostic and statistical manual of mental disorders. (4th ed. Text Revision) (APA,Washington, 2000). - 2. EHLERS A, MAYOU RA, BRYANT B, Behavior Research and Therapy, 41/1 (2003) 1. doi: 10.1016/s0005-7967(01)00126-7. - 3. McNALLY RJ, Trends in cognitive sciences, 10 (2006) 271. doi: 10.1016/j. tics.2006.04.007. - 4. NORIS FN, FRIEDMAN MJ, WATSON PJ, BRYNE CM, DIAZ E, KANIASTY K, Psychiatry, 65 (2002) 207. doi: 10.1521/ psyc.65.3.207.20173. - 5. UDDO M, VASTERLING JT, BRAILEY K, SUTKER PB, J Psychopath Behav Assess, 15 (1993) 43. — 6. NEMEROFF CB, BREMNER JD, FOA EB, MAYBERG H, NORTH CS, STEIN MB, J Psychiatr Res, 40/1 (2006) 20. doi: 10.1016/j.jpsychires.2005.07.005. - 7. BREMNER JD, VYTHILINGAM M, VERMETTEN E, SOUTHWICK SM, MCGLASHAN T, NAZEER A, KHAN S, VACCARINO LV, SOUFER R, GARG PK, NG CK, STAIB LH, DUNCAN JS, CHARNEY DS, Am J Psychiatry, 150 (2003) 924. doi: 10.1176/appi.ajp.160.5.924. - 8. BARRETT LF, TUGADE MM, ENGLE RW, Psychological Bulletin, 130 (2004) 553. doi: 10.1037/0033-2909.130.4.553. - 9. VASTERLING JJ, DUKE LM, BRAILEY K, CONSTANS J, ALLAIN Jr. AN, SUTKER PB, Neuropsychology, 16 (2002) 5. doi: 10.1037//0894-4105.16.1.5. - 10. BLAKE DD, WEATHERS FW, NAGY LM, KALOUPEK D, KLAUMINZER G, CHARNEY DS, KEANE TM, BUCKLEY TC. Clinician-Administered PTSD Scale - CAPS (National Center for PTSD, Instruction Manual, Boston, 2000). - 11. THULSKY D, ZHU J, Wechsler Adult Intelligence Scale WAIS III-R-technical manual (A Harcourt Assessment Company, New York, 1997). - 12. GILBERTSON MW, SHENTON ME, CISZEWSKI A, KASAI K, LASKO NB, ORR SP, PITMAN RK, Nat. Neurosci, 5 (2002) 1242. doi: 10.1038/nn958. - 13. BREMNER JD, SCOTT TM, DELANEY RC, SOUTHWICK SM, MASON JW, JOHNSON DR, INNIS RB, MCCA-
RTHY G, CHARNEY DS, Am J Psychiatry 150 (1993) 1015. doi: 10.1176/ ajp.150.7.1015. PMID: 8317569. — 14. MAZZONNA F. The effect of education on old age health and cognitive abilities (Max Planck Institute, $\mathrm{Mu}$ nich, 2012). - 15. ROCA V, HART J, KIMBRELL T, FREEMAN T, J Neuropsychiatry Clin Neurosci, 18 (2006) 226. doi: 10.1176/ jnp.2006.18.2.226. - 16. YAFFE, K, VITTINGHOFF E, LINDQUIST K, BARNES D, COVINSKY KE, NEYLAN T, KLUSE M, MARMAR C, Arch Gen Psychiatry, 67/6 (2010) 608. doi: 10.1001/archgenpsychiatry.2010.61. - 17. DANCKWERTS A, LEATHEM J, Neuropsychology Review, 13/4 (2013) 221. doi:10.1023/B:NERV.0000009485.76839.b7. - 18. KOVAČIĆKOZARIĆ D, BOROVEĆKI A, Military Medicine, 170/3 (2005) 223. doi: 10.7205/milmed.170.3.223. - 19. YEHUDA R, GOLIER JA, TISCHLER L, HARVEY PD, NEWMARK R, YANG RK, BUCHSBAUM MS, J Psychiatr Res, 41 (2007) 435. doi: 10.1016/j.jpsychires.2005.12.002. - 20. KOSO M, HANSEN S, Eur Psychiatry, 21 (2006) 167. doi: 10.1016/j.eurpsy.2005.06.004. - 21. AMIR N, COLE ME, FOA EB, Cognitive Therapy and Research, 26 (2002) 645. doi.org/10.1023/A:1020309326976. - 22. BREMNER JD, RANDALL P, VERMETTEN E, STAIB L, BRONEN RA, MAZURE C, CAPELLI S, MCCARTHY G, INNIS RB, CHARNEY DS, Biol Psychiatry, 41 (1997) 23. doi: 10.1016/s0006-3223(96)00162-x. — 23. CASSIDY KL, McNALLY RJ, ZEITLIN SB, Cognitive Therapy and Research, 16 (1992) 283. doi.org/10.1007/BF01183282. — 24. REDICK TS, ENGLE RW, The Quarterly Journal of Experimental Psychology, 64 (2011) 1048. doi: 10.1080/17470218.2011.577226. - 25. GURVITS TV, SHENTON ME, HOKAMA H, OHTA H, LASKO NB, GILBERTSON MW, ORR SP, KIKINIS R, JOLESZ FA, MCCARLEY RW, PITMAN RK, Biol Psychiatry, 40 (1996) 1091. doi: 10.1016/S0006-3223(96)00229-6. - 26. KOZARIC-KOVACIC D, HAVELKA MESTROVIC A, RAK D, MUZINIC L, MARINIC I, The Journal of Forensic Psychiatry and Psychology, 24 (2013) 532. doi: 10.1080/14789949.2013.815257.

\section{A. Havelka Meštrović}

Rochester Institute of Technology Croatia, Damira Tomljanovića Gaurana 15, 10000 Zagreb, Croatia

e-mail: ana.havelka-mestrovic@croatia.rit.edu

\section{POSTTRAUMATSKI STRESNI POREMEĆAJ (PTSP) I PROMJENE KOGNITIVNIH FUNKCIJA}

\section{S A Ž E T A K}

Cilj istraživanja bio je dokazati razlike u kognitivnim sposobnostima između bolesnika s PTSP-om i zdravih ispitanika. S obzirom da je PTSP često praćen komorbidnim dijagnozama, u ispitivanje su uključeni bolesnici s PTSP-om koji imaju komorbidnu dijagnozu. Među sudionicima studije bila su 254 hrvatska ratna veterana (60 PTSP i 194 PTSP + komorbiditet) dok su kontrolnu skupinu činili 125 hrvatskih vojnih i civilnih pilota. Dijagnoza PTSP-a procjenjivala se je skalom za ocjenu PTSP-a (CAPS), dok su kognitivne sposobnosti bile mjerene Wechslerovom obavjesnom skalom (WAIS-IIII-R) i Reyevim testom (ROCFT). Rezultati ispitivanja potvrdili su postojanje značajnih razlika u kognitivnim funkcijama između bolesnika s PTSP-om i zdravih ispitanika s obzirom na dob i obrazovanje. Bolesnici s PTSP-om (samo PTSP i PTSP + komorbiditet) pokazali su smanjene opće intelektualne sposobnosti, smanjeni kapacitet radne, numeričke i vizualne memorije te smanjene izvršne funkcije u usporedbi sa zdravim kontrolnim ispitanicima. Ti su rezultati dodatni doprinos boljem razumijevanju i definiranju promjena kognitivnih funkcija te boljem terapijskom pristupu PTSPu kao posljedice traumatskog stresa. 
